Classification

Physics Abstracts

$21.60 \mathrm{E}$

\title{
SUR LES LIMITES D'APPLICATION DU MODÈLE DE STEPHENS
}

\author{
A. ISSA $(*)$ et R. PIEPENBRING \\ Institut des Sciences Nucléaires, BP 257, 38044 Grenoble, France \\ (Reçu le 17 juin 1977, révisé le 13 juillet 1977, accepté le 21 juillet 1977)
}

\begin{abstract}
Résumé. - Les conséquences de l'hypothèse de la conservation 'de $j$ dans le modèle de Stephens sont analysées. Les conditions de l'applicabilité de cette hypothèse dans le cas des sous-couches isolées (l'extension est faite à $N=4$ et $N=3$ ) sont discutées et des explications en sont données. Dans les autres cas, un traitement exact des états propres du mouvement intrinsèque permet néanmoins l'extension du modèle de Stephens sans élargissement de la base de diagonalisation.
\end{abstract}

\begin{abstract}
The limits of the rotation alignment model of Stephens are studied. The conditions of applicability of the assumption of constant $j$ for a unique parity isolated sub-shell (extended to $N=4$ and $N=3$ ) are discussed and explanations are given. A correct treatment of the eigenstates of the intrinsic motion allows however a simple extension of the model to non-isolated sub- shells without enlarging the basis of diagonalisation.
\end{abstract}

1. Introduction. - Dans un récent article de revue [1] Stephens a fait une étude détaillée des effets de Coriolis dans les noyaux et montré que dans certaines conditions on pouvait observer un alignement des particules de valence sur la rotation du cœur.

Dans les noyaux de masse impaire, la particule célibataire peut présenter un tel alignement lorsque, pour une déformation considérée, la surface de Fermi $\lambda$ est située du côté de l'orbite $\Omega=1 / 2$ issue d'une sous-couche sphérique $\left.{ }^{1}\right)$ de $j$ élevé. Ces conditions sont particulièrement favorables dans le cas où ces sous-couches sphériques sont les seules $\left({ }^{2}\right)$ à avoir une parité donnée dans une certaine zone d'énergie. C'est le cas des sous-couches $h_{11 / 2}, i_{13 / 2}$ et $\mathrm{j}_{15 / 2}$ respectivement dans les couches $N=5,6$ et 7 .

On peut alors, avec une bonne approximation [1], résoudre l'équation de Schrödinger en admettant que $j$ est un bon nombre quantique. Avec une telle hypothèse :

a) les énergies de particules dans un potentiel de Nilsson dépendent linéairement de la déformation $\beta$ du noyau;

$b$ ) les éléments de matrice des opérateurs $j_{ \pm}$et en particulier les facteurs de découplage $a$, ne dépendent pas de la déformation.

(*) Boursier du Conseil National pour la Recherche Scientifique, Beyrouth, Liban.

( $\left.{ }^{1}\right)$ Nous conviendrons d'utiliser le terme sous-couche pour désigner une état sphérique et le terme orbite pour désigner un état déformé.

$\left({ }^{2}\right)$ Dans la suite, nous conviendrons de les désigner par souscouches isolé(').
Il nous a d'abord semblé utile de tester la valeur de cette approximation dans les cas où on s'attend à trouver un alignement sur la rotation. D'une manière plus générale, nous examinerons, dans quelle mesure et comment ce phénomène se modifie pour des états issus de sous-couches ayant des valeurs de $j<11 / 2$. Un cas particulier intéressant est celui où deux souscouches avec $j$ et $j-1$ apparaissent énergétiquement voisines mais globalement isolées des autres (cas des sous-couches $h_{9 / 2}$ et $\mathrm{f}_{7 / 2}(N=5)$ d'une part et $\mathrm{g}_{7 / 2}$ et $\mathrm{d}_{5 / 2}(N=4)$ d'autre part).

Après avoir rappelé les éléments nécessaires à la compréhension du phénomène étudié, nous comparerons les résultats des calculs utilisant ou non les approximations $a$ ) et $b$ ) pour certaines orbites à $j$ élevés, l'hamiltonien du système étant diagonalisé dans une base tronquée ne faisant intervenir que les niveaux déformés issus de la sous"couche sphérique considérée. Enfin, on étudiera les deux cas cités plus haut où cette base est élargie à deux sous-couches voisines.

2. Rappel. - Pour étudier les effets de Coriolis dans un noyau de masse impaire, on considère [2] le système nucléaire formé par une particule liée à un cœur rigide, déformé, à symétrie axiale et qui tourne autour d'un axe perpendiculaire à l'axe de symétrie. L'hamiltonien de ce système s'écrit :

$$
\begin{aligned}
\mathscr{H}=\mathscr{H}_{\mathrm{p}}+\frac{\hbar^{2}}{2 \mathfrak{J}}[I(I+1)- & \left.K^{2}\right]+\mathscr{H}_{\mathrm{c}}+ \\
& +\frac{\hbar^{2}}{2 \mathfrak{J}}\left[\left\langle j^{2}\right\rangle-\Omega^{2}\right]
\end{aligned}
$$


où $\mathcal{H}_{\mathrm{p}}$ est 1 'hamiltonien décrivant le mouvement individuel de la particule célibataire (celui de Nilsson [3] par exemple), $J$ est le moment d'inertie du cœur, $I$ et $j$ sont respectivement les moments angulaires du noyau et de la particule, $K$ et $\Omega$ sont leurs projections respectives sur l'axe de symétrie. Dans le cas qui nous intéresse $K=\Omega$. $\mathscr{H}_{\mathrm{c}}$ est le terme du couplage de Coriolis dont les éléments de matrice sont :

$$
\begin{aligned}
& \left\langle I, \Omega \pm 1\left|\mathcal{H}_{\mathrm{c}}\right| I, \Omega\right\rangle= \\
& =-\frac{\hbar^{2}}{2 J} \sqrt{(I \mp K)(I \pm K+1)}\left\langle\Omega \pm 1\left|j_{ \pm}\right| \Omega\right\rangle
\end{aligned}
$$

où l'élément de matrice $\left\langle\Omega \pm 1\left|j_{ \pm}\right| \Omega\right\rangle$, dans l'hypothèse de $j$ pur, peut s'écrire :

$$
\left\langle j, \Omega \pm 1\left|j_{ \pm}\right| j, \Omega\right\rangle=\sqrt{(j \mp \Omega)(j \pm \Omega+1)} .
$$

Avec la même hypothèse, les énergies individuelles dans un potentiel de Nilsson sont données par :

$$
e_{\Omega}=\varepsilon_{j}^{\prime}+k \beta\left[\frac{3 \Omega^{2}-j(j+1)}{4 j(j+1)}\right]=\varepsilon_{j}+C \Omega^{2}
$$

où $\mathcal{E}_{j}^{\prime}$ et $\mathcal{E}_{j}$ ne dépendent pas de $\Omega$ et où $\beta$ est le paramètre de déformation introduit par Bohr [2] lié au paramètre $\varepsilon$ introduit par Lamm [4] par la relation :

$$
\beta=\sqrt{\frac{4 \pi}{5}} \frac{6 \varepsilon-\varepsilon^{2}}{9+2 \varepsilon^{2}} .
$$

Enfin, le dernier terme de l'éq. (1) est ce qu'on appelle [5] le terme de recul, il sera introduit dans tous nos calculs.

Lorsqu'on tient compte des corrélations de paires, il faut remplacer l'énergie des particules $e_{\Omega}$ par l'énergie des quasi-particules :

$$
E(\Omega)=\sqrt{\left(e_{\Omega}-\lambda\right)^{2}+\Delta^{2}}-\Delta
$$

où $\lambda$ désigne la surface de Fermi et $2 \Delta$ le gap. Un facteur multiplicatif $\left(u_{\Omega} v_{\Omega+1}+u_{\Omega+1} v_{\Omega}\right)$ s'introduit également dans les éléments de matrice de Coriolis (3).

Pour limiter au maximum le nombre de paramètres [1] la constante d'inertie $A=\hbar^{2} / 2 \mathfrak{J}$ est supposée liée au paramètre de déformation $\beta$ par la relation empirique introduite par Grodzins [6] :

$$
\frac{\hbar^{2}}{2 J}=\frac{1225}{6 A^{7 / 3} \beta^{2}} \mathrm{MeV}
$$

où $\mathcal{A}$ est la masse du noyau. D'autre part, $\Delta$ est calculé par la relation suivante [7] :

$$
\Delta=\frac{135}{\mathfrak{A}}
$$

qui permet de retrouver correctement les difference de masse pair-impair dans la région $100<.1=2(1)$

A l'aide des éqs. (2) à (8) et pour une sous ، uch. donnée $j$, on peut diagonaliser l'hamiltonien de l'éq. (1) pour toute valeur de $I, \lambda$ et $\beta$ (sauf pour $\beta=0$ qu'on atteint par l'approche des petites valeurs de $\beta$ ).

Remarquons que les approximations $a$ ) et $b$ ) sont introduites par les formules (4) et (3) respectivement.

3. Cas d'une seule sous-couche. - Pour chaque sous-couche, nous avons effectué deux calculs : le premier est celui de Stephens décrit au paragraphe précédent, le second (que nous appellerons exact $\left({ }^{3}\right)$ dans la suite) lève les approximations des relations (3) et (4) : les énergies individuelles $e_{\Omega}$ et les éléments de matrice de $j_{ \pm}$sont calculés proprement dans le cadre du modèle de Nilsson comme explicité, par exemple, dans la référence [8].

Dans les deux calculs, l'hamiltonien (1) est diagonalisé pour chaque valeur de $I$ et $\beta$ dans une base tronquée ne comprenant que les orbites déformées issues de la sous-couche étudiée. La matrice $\mathcal{H}$ est d'ordre $I+1 / 2$ pour $I<j$ et $j+1 / 2$ pour $I \geqslant j$.

3.1 SOUS-COUCHES ISOLÉES. - Nous allons illustrer ce cas en considérant la sous-couche $\mathrm{h}_{11 / 2}(N=5)$ pour des noyaux à nombre de neutrons $\mathcal{N}$ impair. Les résultats des deux calculs correspondants sont comparés dans la figure 1 où la surface de Fermi est choisie complètement en dessous de la sous-couche. L'accord entre les deux calculs est spécialement bon lorsque $\lambda$ est placé au voisinage de l'orbite $\Omega=j=11 / 2$ c'est-à-dire pour $\varepsilon<0$ (ou pour $\varepsilon>0$ lorsque $\lambda$ est au-dessus de la sous-couche), on observe une bande rotationnelle bien développée basée sur $\Omega=11 / 2$ à partir de $\varepsilon \simeq-0,15$. Lorsque $\lambda$ est placé près de l'orbite $\Omega=1 / 2(\varepsilon>0)$, les deux calculs font apparaître de petites différences caractéristiques :

- les niveaux de la bande découplée ou alignée sur la rotation, tout en conservant des écartements voisins des niveaux du cœur, ont des énergies variant plus rapidement avec $\varepsilon$ dans le calcul exact. Ceci est aussi le cas des niveaux ayant $I=j-2, j-4$ etc.,

- pour une certaine valeur de $\varepsilon$ (de l'ordre de 0,30 pour le cas de la sous-couche $h_{11 / 2}$ considérée) la quantité $\frac{\partial(\Delta E(I))}{\partial \varepsilon}$ change de signe pour les niveaux ayant $I+1 / 2$ impair dans le calcul exact, alors que dans le calcul de Stephens la variation des énergies $\Delta E(I)$ de ces niveaux est monotone.

D'autre part, nous avons regardé si la symétrie prolate-oblate observée par Stephens [1] était conservée dans le calcul exact. Pour ce faire, nous avons fait des calculs pour deux positions de la surface de Fermi $\lambda$, équidistantes, et de part et d'autre, de la position de la sous-couche $\mathrm{h}_{11 / 2}$ pour $\varepsilon=0$. Il

$\left(^{3}\right)$ Des diagonalisations « exactes» de l'hamiltonien (1) ont également été effectuées par d'autres auteurs [12]. 




Fig. 1. - Résultats de la diagonalisation de l'éq. (1) pour différentes valeurs du paramètre de déformation, dans le cas de la sous-couche $h_{11 / 2}$. Pour chaque spin $I$, l'état le plus bas en énergie est représenté. Les courbes continues correspondent au calcul exact et les courbes discontinues au calcul de Stephens. En ordonnée, nous portons l'énergie de l'état de spin $I$ moins celle de l'état de spin 11/2 de plus basse énergie en unité $E_{2+}$. En abscisse, nous portons le paramètre $\varepsilon$. Pour $\varepsilon<0$ les deux calculs donnent des résultats très proches et pour ne pas encombrer la figure nous ne représentons que les résultats correspondant au calcul exact. La surface de Fermi est $\lambda=5,314 \hbar \omega_{0}$. La masse nucléaire est : $\mathfrak{A}=140, \mathcal{N}$ est impair.

[Results of diagonalizing eq. (1) for an $h_{11 / 2}$ orbital at various values of the deformation parameter $\varepsilon$. For each spin $I$ the lowest state is shown. The full lines correspond to exact calculations, the dashed lines correspond to Stephens calculations. The ordinate is the difference between the eigenvalues and that of the lowest $I=11 / 2$ state, in units of $E_{2+}$. For $\varepsilon<0$ we show the results of exact calculations only because both calculations give the same results approximately. The Fermi surface $\lambda$ is $5.314 \hbar \omega_{0}$. Nuclear mass is $\mathcal{A}=140, \mathcal{N}$ is odd.]

apparaît que cette symétrie existe pour les niveaux de la bande rotationnelle $\Omega=j$ et pour les niveaux de la bande découplée. Elle est cependant légèrement détruite pour les niveaux de spin $I=j \pm 2 p+1$.

Toutes les remarques du paragraphe précédent concernant la sous-couche $h_{11 / 2}$ dans le cas des noyaux à $\mathcal{N}$ impair $\left(\mathcal{A} \simeq 140:{ }^{133} \mathrm{Ce}_{75},{ }^{135} \mathrm{Nd}_{75}, \ldots\right.$ et $\left.\mathcal{A} \simeq 170:{ }^{169} \mathrm{Yb}_{99},{ }^{171} \mathrm{Hf}_{99}, \ldots\right)$ sont aussi valables dans le cas des noyaux à nombre de protons 3 impair $\left(\mathcal{A} \simeq 190:{ }_{77}^{199} \mathrm{I}_{r} \ldots\right.$ et $\left.\mathcal{A} \simeq 200:{ }^{199} \mathrm{Tl}, \ldots\right)$; elles le


$\mathrm{j}_{15 / 2}$ (transuraniens) pour des noyaux à $\mathcal{N}$ impair.

Dans le but de rechercher d'autres sous-couches avec $j<11 / 2$ où le modèle de Stephens peut s'appliquer, nous avons étudié les sous-couches isolées $\mathrm{g}_{9 / 2}(N=4)$ et $\mathrm{f}_{7 / 2}(N=3)$ et obtenu des résultats analogues aux précédents. Il semble donc qu'on puisse appliquer le modèle de Stephens à toutes les souscouches isolées pour $N \geqslant 3$ même si le nombre quantique $j$ est moins grand.

Dans le paragraphe suivant, nous traitons un cas typique où la sous-couche n'est plus isolée énergétiquement c'est le cas, par exemple, de l'une des souscouches $h_{9 / 2}$ et $f_{7 / 2}(N=5)$ ou de l'une des souscouches $\mathrm{g}_{7 / 2}$ et $\mathrm{d}_{5 / 2}(N=4)$. Pour distinguer celles-ci des sous-couches isolées nous conviendrons de les désigner par sous-couches voisines dans la suite.

3.2 Sous-COUCHES voisines. - Ce cas est illustré par l'étude de la sous-couche $h_{9 / 2}$. Ici les choses ne sont plus identiques pour les cas des noyaux à $\mathcal{N}$ ou 3 impairs comme cela l'a été dans les sous-couches isolées, c'est pourquoi nous traitons les deux cas séparément.

3.2.1 Cas 3 impair. - Les résultats concernant ce cas sont représentés sur la figure 2 où $\lambda$ est choisi

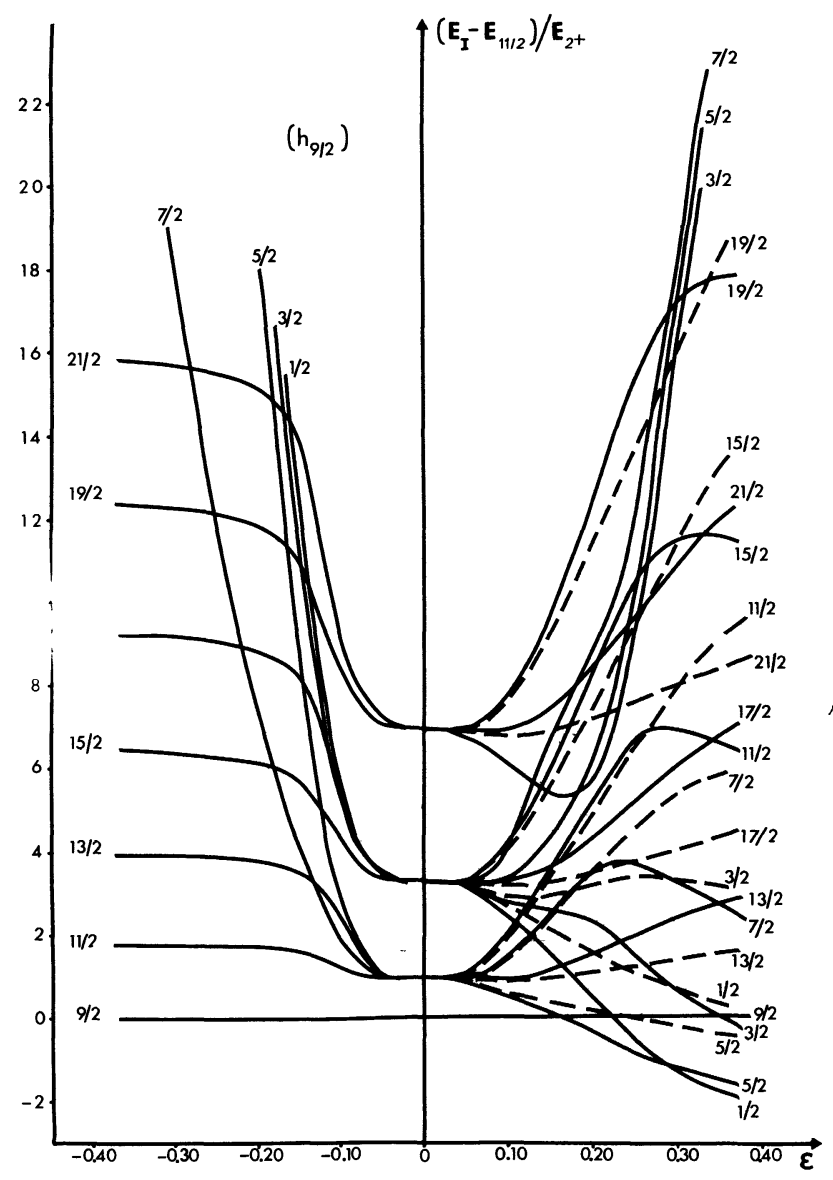

Fig. 2. - Même chose que dans la figure 1 mais ici dans le cas de la sous-couche $h_{9 / 2}, \lambda=5,80 \hbar \omega_{0}, \mathcal{A}=190$ et $Z$ impair.

[The same as figure 1 but for an $h_{9 / 2}$ orbital, $\lambda=5.80 \hbar \omega_{0}$. $\mathcal{A}=190$, $Z$ odd.] 
en dessous de la sous-couche $h_{9 / 2}$. L'accord entre les deux calculs (Stephens et exact) est toujours bon du côté où $\lambda$ est au voisinage de l'orbite $\Omega=j=9 / 2$ $(\varepsilon<0)$. Lorsque $\lambda$ est placé du côté de l'orbite $\Omega=1 / 2(\varepsilon>0)$ on peut faire les remarques suivantes :

- les niveaux de la bande découplée, de spin $I=j+2, j+4, \ldots$, ont des énergies plus petites avec le calcul de Stephens qu'avec le calcul exact où leurs séparations ne sont plus tout à fait celles du cœur ;

- par contre, les énergies des niveaux de petit spin $(I=1 / 2,3 / 2,5 / 2)$ sont plus basses avec le calcul exact (comme dans le cas des sous-couches isolées);

- enfin, dans les deux calculs les niveaux de spin $I=j+2 p+1$ ont des énergies comparables lorsque $\varepsilon \lesssim 0,25$ c'est vers cette déformation que $\frac{\partial(\Delta E(I))}{\partial \varepsilon}$ change de signe.

Lorsque $\lambda$ est placé au-dessus de la sous-couche $h_{9 / 2}$, les mêmes constatations sont valables, seulement ici une bande $3 / 2$ non découplée apparaît (pour $\varepsilon<0$ ) dans une gamme d'énergie assez basse et cela uniquement avec le calcul exact.

3.2.2 Cas $\mathcal{N}$ impair. - Avant de discuter ce cas il faut noter que dans le calcul de Stephens on ne dis-

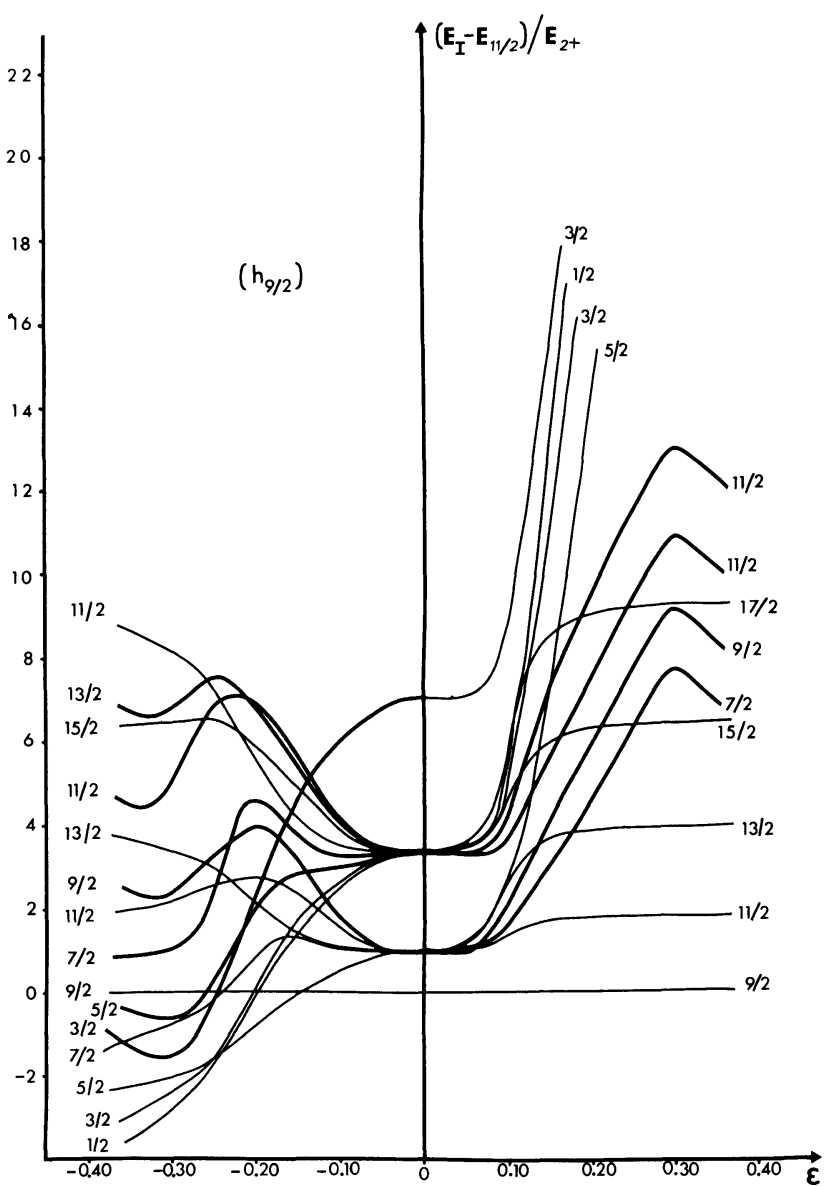

FIG. 3. - Même chose que dans la figure $1, \lambda=7,20 \hbar \omega_{0}$, $\mathcal{A}=173$ et $\mathcal{N}$ impair.

[The same as figure $2, \lambda=7.20 \hbar \omega_{0}, \mathcal{A}=173 . \mathcal{N}$ odd.] tingue pas entre protons et neutrons, cela est dû à l'introduction des approximations $a$ ) et $b$ ) ; par conséquent, ce calcul donne dans le cas $\mathcal{N}$ impair des résultats identiques au cas 3 impair.

Aussi, nous montrons uniquement les résultats du calcul exact sur la figure 3 où $\lambda$ est choisi au-dessus de la sous-couche $h_{9 / 2}$. On constate des différences importantes avec le calcul de Stephens :

- d'abord du côté oblate $(\varepsilon<0)$ on n'a plus la bande découplée basée sur $\Omega=9 / 2$ et les séparations en énergie entre les niveaux de spin $I=j, j+2$, $j+4, \ldots$ s'écartent considérablement de celles du cœur.

De plus, on voit se former une bande $1 / 2$ non découplée pour $\varepsilon \lesssim-0,25$. Outre la bande $1 / 2$ on observe, pour $\varepsilon \lesssim-0,23$, une bande $3 / 2$ non découplée dans la même zone d'énergie que la bande $1 / 2$. C'est un fait nouveau par rapport au calcul de Stephens, qu'on retrouve également pour les souscouches $\mathrm{f}_{7 / 2}(N=5) \mathrm{g}_{7 / 2}$ et $\mathrm{d}_{5 / 2}(N=4)$;

- du côté prolate $(\varepsilon>0)$ on observe une bande rotationnelle $9 / 2$ en bon accord avec le calcul de Stephens, mais en plus on observe une bande $7 / 2$ très rapprochée de la bande $9 / 2$ par rapport à ce que donne le calcul de Stephens.

Lorsqu'on place $\lambda$ en dessous de la sous-couche $\mathrm{h}_{9 / 2}$ et symétriquement par rapport à la position de $\mathrm{h}_{9 / 2}$ pour $\varepsilon=0$ on n'observe plus les bandes $3 / 2$ et $7 / 2$ dans les mêmes zones d'énergie que plus haut mais on observe toujours la bande $1 / 2$ non découplée du côté prolate $(\varepsilon>0)$.

Nous constatons donc que la symétrie prolateoblate qui est une conséquence de l'approximation $a$ ) dans le calcul de Stephens, est complètement détruite dans le calcul exact effectué avec les énergies et les fonctions d'ondes des états de Nilsson. Ceci peut en fait être prévu : en effet, sur la figure 4 où sont représentées les énergies individuelles des états de Nilsson en fonction de la déformation $\varepsilon$, on peut constater le rapprochement considérable $(\varepsilon<0)$ des orbites $1 / 2-[530]$ et $3 / 2-$ [521] qui ne ressemblent en rien aux droites données par l'approximation $a$ ) de Stephens (formule (4)). Ce sont sur ces orbites que sont construites les bandes $1 / 2$ et $3 / 2$ observées dans le cas $\mathcal{N}$ impair. De même, pour $0,05 \lesssim \varepsilon \lesssim 0,15$ on voit un pseudo-croisement des deux courbes représentant les orbites $7 / 2-$ [503] et $7 / 2-$ [514] qui a pour effet de les écarter l'une de l'autre tout en rapprochant la première de l'orbite $9 / 2-$ [505], alors qu'avec l'approximation a) de Stephens et pour $\varepsilon \gtrsim 0,15$ l'orbite $7 / 2-$ [503] deviendrait l'orbite $7 / 2-[514]$ et inversement. Cela explique l'apparition des bandes 7/2 et 9/2 rapprochées dans le calcul exact.

Les pseudo-croisements des orbites de même nombre quantique $\Omega$ mais issues de sous-couches différentes sont à l'origine de l'observation de plusieurs bandes dans une même zone de basse énergie. Ainsi ce phénomène se reproduit pour les sous-couches 


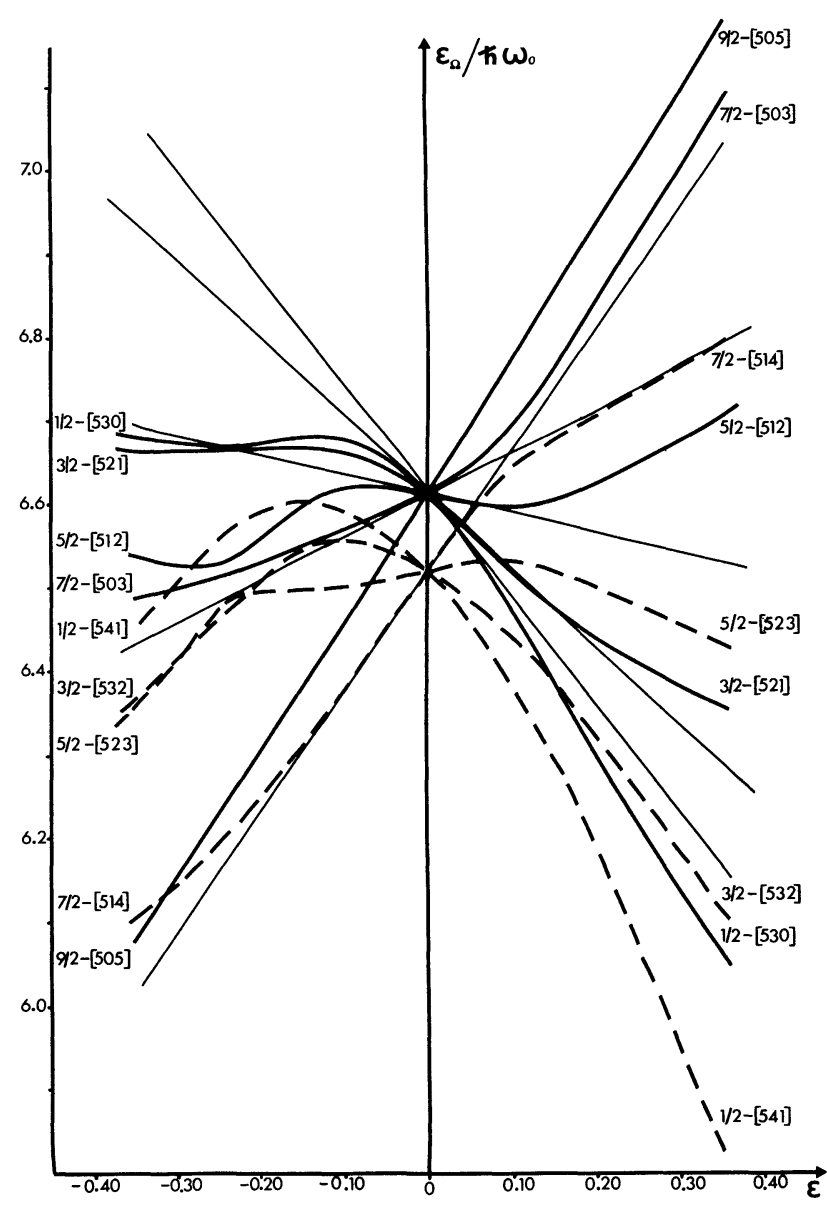

Fig. 4. - Energies individuelles des états de Nilsson (en unité $\left.\hbar \omega_{0}\right)$ en fonction de la déformation. Les courbes en gros caractère continues et discontinues représentent les orbites issues respectivement de la sous-couche $h_{9 / 2}$ et de la sous-couche $f_{7 / 2}$. Les courbes continues en petit caractère sont les droites utilisées par Stephens et qui représentent les orbites issues de la sous-couche $\mathrm{h}_{9 / 2}$.

[Single particle energies of the Nilsson states (in units of $\hbar \omega_{0}$ ) as a function of $\varepsilon$. The dark lines, full and dashed, are $h_{9 / 2}$ and $f_{7 / 2}$ orbitals respectively. The other lines are the street lines used by Stephens for an $h_{9 / 2}$ orbital.]

$\mathrm{f}_{7 / 2}(N=5) \mathrm{g}_{7 / 2}$ et $\mathrm{d}_{5 / 2}(N=4)$ où dans le cas des noyaux à $\mathcal{N}$ impair et lorsque $\lambda$ est au-dessus de la sous-couche, on observe même trois bandes $1 / 2$, $3 / 2$ et $5 / 2$ dans une même zone d'énergies (pour $\varepsilon<0$ ).

Un phénomène inverse se produit dans le cas de la sous-couche $\mathrm{g}_{7 / 2}$ pour les noyaux à $\mathcal{N}$ impair mais où $\lambda$ est placé en dessous de la sous-couche $\mathrm{g}_{7 / 2}$, on observe alors (pour $\varepsilon>0$ ) une bande $3 / 2$ mais cette fois-ci avec le calcul de Stephens seulement. C'est aussi une conséquence de deux pseudo-croisements qui séparent les orbites $1 / 2+[420]$ et $3 / 2+[411]$ de la sous-couche $\mathrm{g}_{7 / 2}$, alors qu'elles sont très rapprochées l'une de l'autre avec la formule (4) de Stephens.

Ces effets de pseudo-croisements, qui n'existent pas dans les sous-couches isolées, ont des conséquences importantes dans le traitement des sous-couches « voisines ».

\subsection{QUELQUES REMARQUES GÉNÉRALES CONCERNANT} LE CAS D'UNE SEULE SOUS-COUCHE. - Les facteurs de découplage $a$ calculés à partir des fonctions d'onde des états de Nilsson issus de différentes sous-couches, sont représentés en fonction de la déformation $\varepsilon$ sur la figure 5 . Sur celle-ci on distingue très nette-

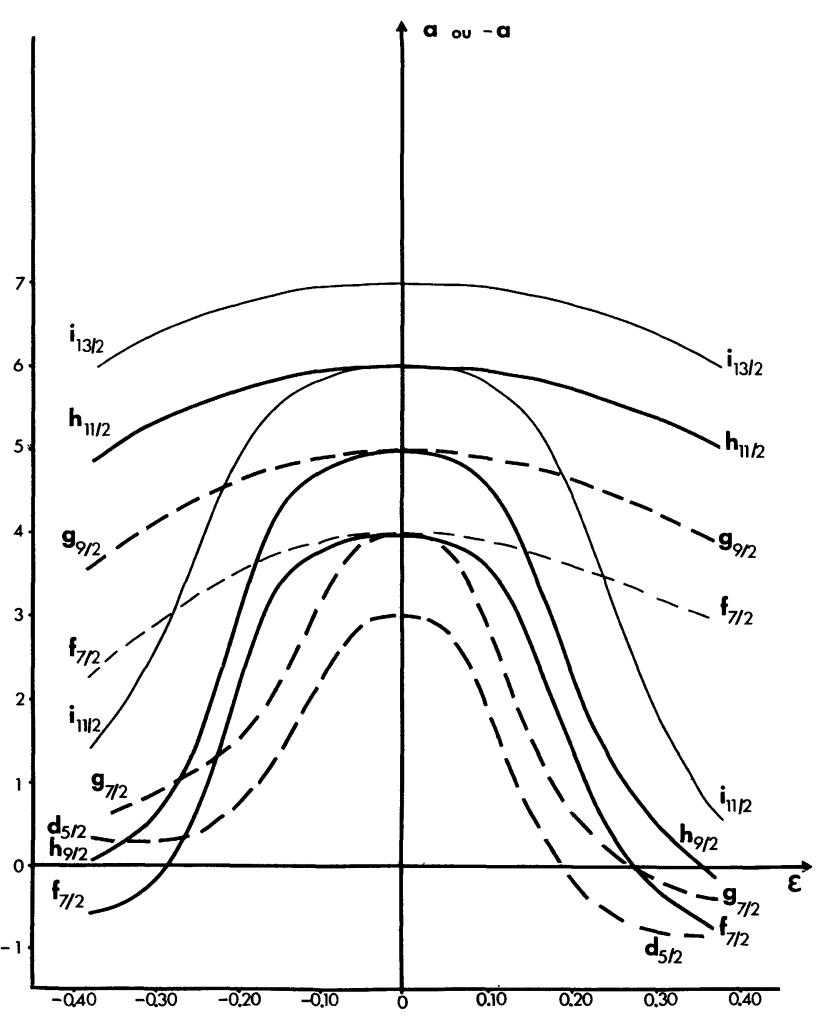

Fig. 5. - Les facteurs de découplage $a$ calculés à partir des fonctions d'onde des états de Nilsson issus des différentes souscouches, en fonction de $\varepsilon$.

[The decoupling parameters calculated with the wave functions of the Nilsson states for different orbitals as a function of $\varepsilon$.]

ment une classe de courbes qui varient peu avec $\varepsilon$, elles correspondent aux sous-couches isolées. Les autres courbes, correspondant aux sous-couches non isolées, varient beaucoup plus avec $\varepsilon$. On est ainsi amené à penser que le succès du modèle de Stephens pour les premières pourrait être dû au fait que le facteur de découplage est pratiquement constant avec $\varepsilon$. C'est ce que nous essayons de montrer avec la sous-couche $h_{9 / 2}$ sur la figure 6 où sont représentés les résultats de deux calculs : d'une part le calcul exact, de l'autre un calcul qui ne comporte que les termes diagonaux :

$$
\begin{aligned}
E(I)=\mathcal{H}_{\mathrm{p}}(\Omega) & +\frac{\hbar^{2}}{2 J} \times \\
\times & \times\left[I(I+1)+(-)^{I+1 / 2}(I+1 / 2) a\right]
\end{aligned}
$$

où $a$ est calculé exactement (Fig. 5) à l'aide des fonctions d'onde des états de Nilsson. On constate que pour des déformations $\varepsilon \gtrsim 0,26$ les deux calculs 
(Fig. 6) sont comparables et donnent pratiquement une bande $1 / 2$ non découplée. Ceci peut s'expliquer par le fait (Fig. 5) que pour $\varepsilon \simeq 0,26$ le facteur de découplage $a\left(\mathrm{~h}_{9 / 2}\right)$ est de $1 / 5$ de sa valeur pour $\varepsilon=0$ (valeur adoptée par Stephens pour tout $\varepsilon$ ) alors que les autres éléments de matrice de $j_{+}$(Fig. 8) conservent encore $80 \%$ de leur valeur pour $\varepsilon=0$ (sauf $\langle 9 / 2|j+| 7 / 2\rangle$ qui en est à $25 \%$ ).



Fig. 6. - En ordonnée sont portés les résultats de la diagonalisation de l'éq. (1) obtenus avec le calcul exact et les résultats de la diagonalisation de l'éq. (9) pour différentes valeurs de $\varepsilon$. Les courbes en gros caractère représentent les premiers et les courbes en petit caractère représentent les seconds.

[Results of diagonalizing eq. (1) obtained with exact calculations and results of diagonalizing eq. (9) at various values of $\varepsilon$. The dark lines represent the former case, the other represent the latter.]

Nous avons par ailleurs testé la pureté en $j$ de chaque orbite considérée en calculant $\left|C_{j l}^{\alpha \Omega}\right|^{2}$ où les $C_{j l}^{\alpha S 2}$ sont les composantes des états de Nilsson sur la base sphérique $|N l j\rangle$

$$
\chi_{\Omega}^{\alpha}=\sum_{j l} C_{j l}^{\alpha \Omega}|N l j\rangle
$$

et où $\alpha$ désigne la sous-couche sphérique dont est issu l'état de Nilsson. Un tel calcul montre que pour toutes les orbites issues de sous-couches isolées et pour $|\varepsilon| \lesssim 0,25$ il y a une composante $C_{j l} \gtrsim 0,80$, c'est celle qui correspond au nombre quantique $j$ de la sous-couche isolée. L'hypothèse de $j$ pur qui consiste à prendre cette composante égale à 1 est donc justifiée pour ces sous-couches. Pour les autres sous-couches cette hypothèse est complètement fausse et par conséquent les formules (3) et (4) n'ont aucune justification, il faut considérer toutes les composantes $C_{j l}$ dans le calcul des énergies individuelles et des éléments de matrice des opérateurs $j_{ \pm}$, c'est ce que nous avons fait dans le calcul exact.

4. Cas de deux sous-couches. - Dans le but d'étendre le modèle de Stephens à plusieurs sous-couches, il est intéressant de calculer les éléments de matrice $\left\langle\alpha, \Omega+1\left|j_{+}\right| \alpha^{\prime}, \Omega\right\rangle$ pour des orbites issues de deux sous-couches différentes $\alpha$ et $\alpha^{\prime}$.

Si $\alpha$ (ou $\alpha^{\prime}$ ) est une sous-couche isolée et $\alpha^{\prime}$ (ou $\alpha$ ) une autre sous-couche de la même couche $N \geqslant 3$, le calcul de ces éléments de matrice montre qu'ils sont négligeables par rapport à ceux calculés entre les orbites de la même sous-couche isolée, ce qui est un argument supplémentaire qui justifie l'hypothèse de $j$ pur pour les sous-couches isolées.

Sur les figures 7 et 8 sont représentés les éléments de matrice de $j_{+}$pour lesquels $\alpha \not \equiv \alpha^{\prime}$ (Fig. 7) et $\alpha \equiv \alpha^{\prime}$ (Fig. 8) où $\alpha$ et $\alpha^{\prime}$ désignent l'une ou l'autre des

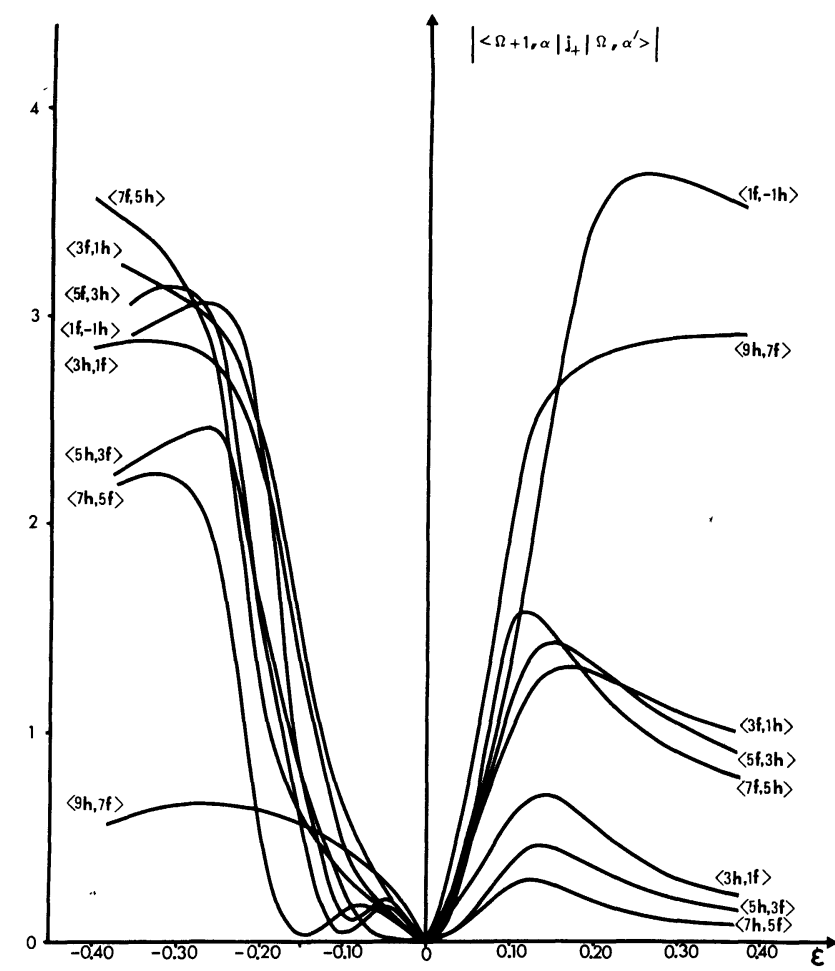

FIG. 7. - Eléments de matrice $\left\langle\Omega+1, \alpha\left|j_{\mp}\right| \Omega, \alpha^{\prime}\right\rangle$, en valeur absolue, calculés entre les états issus de la sous-couche $h_{9 / 2}$ et les états issus de la sous-couche $\mathrm{f}_{7 / 2}$. Nous avons allégé les écritures, par exemple $\langle 5 h, 3 f\rangle$ se lit $:\left|\left\langle 5 / 2, h_{9 / 2}\left|j_{+}\right| 3 / 2, f_{7 / 2}\right\rangle\right|$.

[Absolute values of matrix elements $\left\langle\Omega+1, \alpha\left|j_{+}\right| \Omega, \alpha^{\prime}\right\rangle$ calculated between states issued from $h_{9 / 2}$ and $f_{7 / 2}$ orbitals. We have simplified the notation, for example $\langle 5 h, 3 f\rangle$ denotes :

$$
\left.\left|\left\langle 5 / 2, h_{9 / 2}\left|j_{+}\right| 3 / 2, f_{7 / 2}\right\rangle\right| .\right]
$$




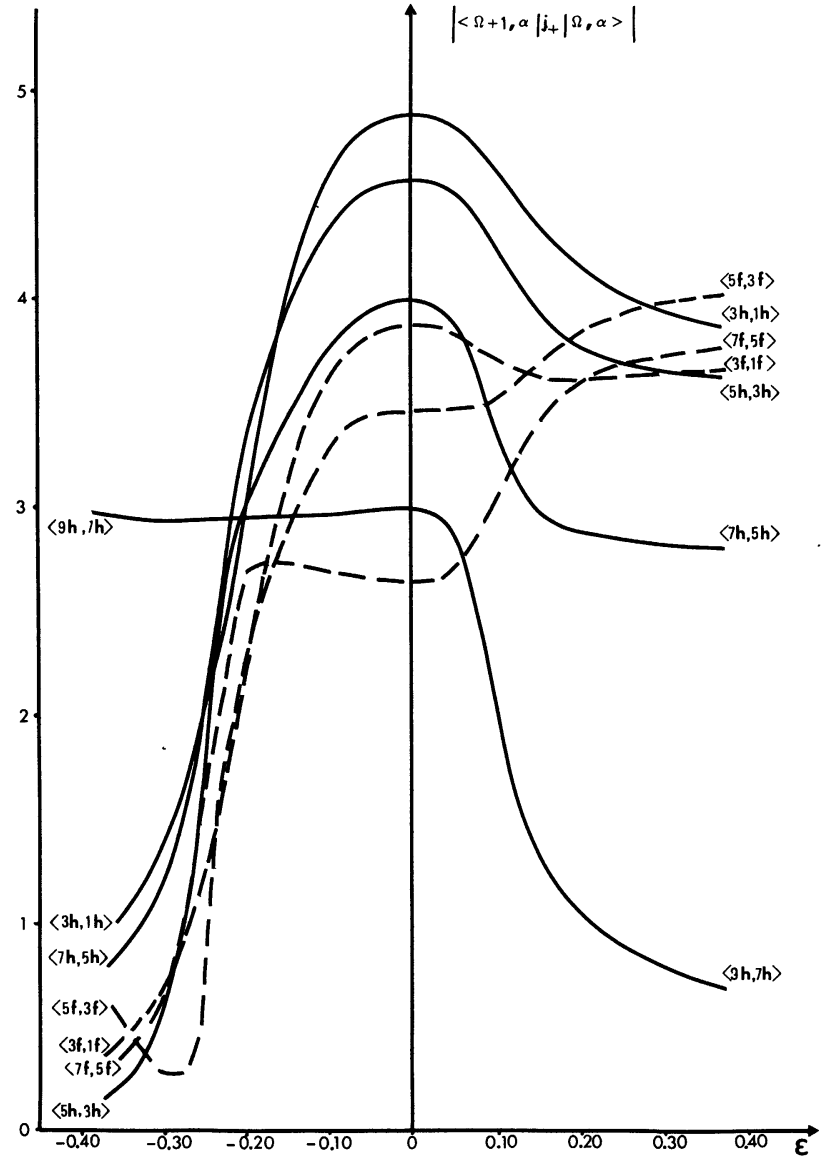

FIG. 8. - Eléments de matrice $\left\langle\Omega+1, \alpha\left|j_{+}\right| \Omega, \alpha\right\rangle$, en valeur absolue, calculés entre les états issus de la même sous-couche. Les courbes continues correspondent à la sous-couche $h_{9 / 2}$ et les courbes discontinues correspondent à la sous-couche $f_{7 / 2}$. La même notation que dans la figure 8 a été utilisée ici.

[Absolute values of matrix elements $\left\langle\Omega+1, \alpha\left|j_{+}\right| \Omega, \alpha\right\rangle$ calculated between states issued from the same orbital. Full lines correspond to an $h_{9 / 2}$ orbital and dashed lines correspond to an $f_{7 / 2}$ orbital. The same notation as in figure 7 is used here.]

sous-couches $\mathrm{h}_{9 / 2}$ et $\mathrm{f}_{7 / 2}(N=5)$. La comparaison des deux montre que pour certaines déformations les premiers sont du même ordre de grandeur que les seconds. D'autre part, les éléments de matrice calculés entre les orbites issues d'une des sous-couches $\alpha$ ou $\alpha^{\prime}$ d'une part et les orbites issues des autres souscouches $N=5\left(\mathrm{~h}_{11 / 2}, \mathrm{f}_{5 / 2}, \mathrm{p}_{3 / 2}\right.$ et $\left.\mathrm{p}_{1 / 2}\right)$ d'autre part, sont très petits comparés à ceux des figures 7 et 8 . On peut ainsi espérer, qu'en incluant les éléments de matrice entre les orbites des deux sous-couches $h_{9 / 2}$ et $f_{7 / 2}$, traiter proprement l'ensemble de ces deux sous-couches séparément des autres sous-couches $N=5$. On observe une situation analogue pour l'ensemble des deux sous-couches $\mathrm{g}_{7 / 2}$ et $\mathrm{d}_{5 / 2}(N=4)$.

Le traitement d'un tel ensemble de deux souscouches peut se faire d'une manière analogue au cas d'une seule sous-couche, la base étant simplement élargie aux deux sous-couches considérées.

Si dans les calculs concernant une seule souscouche on peut prendre les valeurs usuelles [4] du paramètre $\mu$ du potentiel de Nilsson $(\mu=0,42$ pour un champ neutron et $\mu=0,60$ pour un champ proton dans la région des terres rares), le choix de $\mu$ joue un rôle fondamental dans le traitement d'un ensemble de deux sous-couches. C'est en effet de ce choix que dépend l'ordre respectif des sous-couches et leur écartement en énergie (Fig. 9).

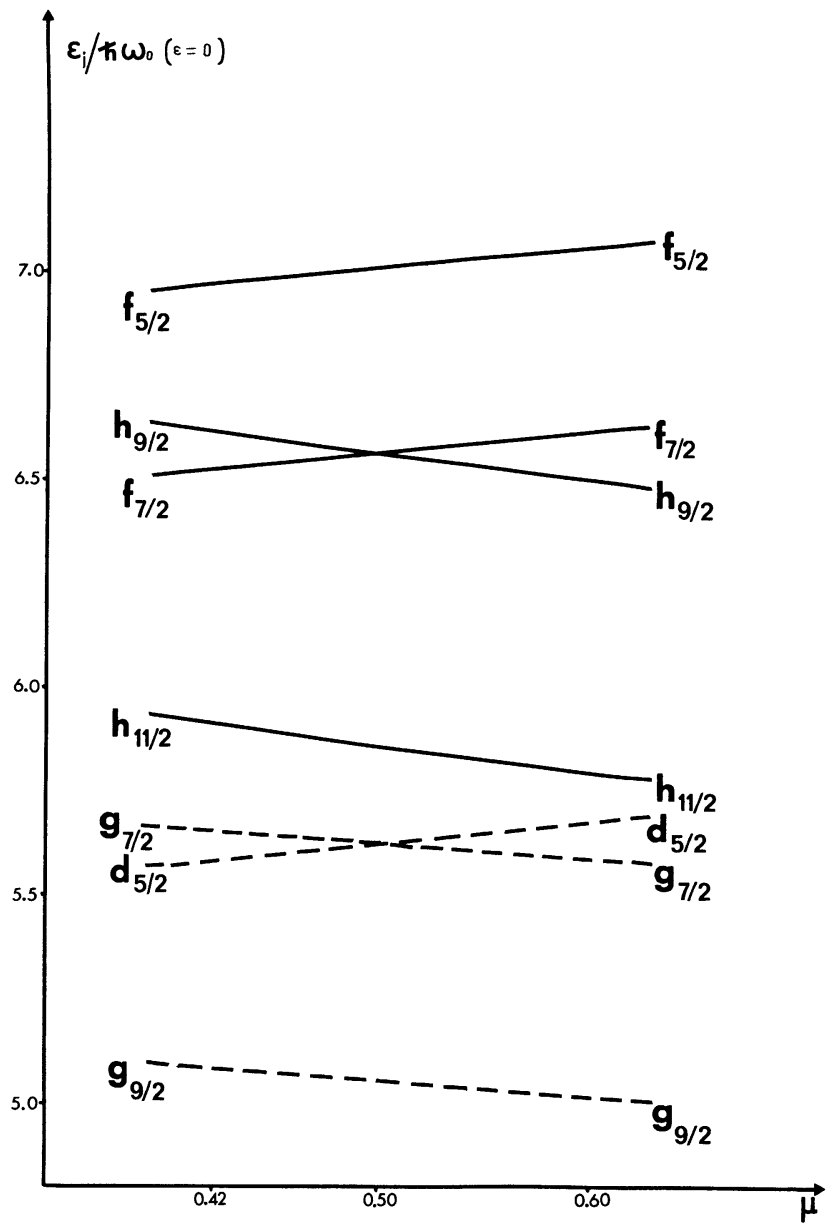

Fig. 9. - Variations en fonction du paramètre $\mu$ du potentiel de Nilsson, de l'énergie (en unité $\hbar \omega_{0}$ ) de quelques sous-couches (couches $N=5$ et $N=4$ ) à déformation nulle $\varepsilon=0$.

[The energies (in units of $\hbar \omega_{0}$ ) of some orbitals (shells $N=5$ and $N=4$ ) for $\varepsilon=0$ as a function of the parameter $\mu$ of the Nilsson potential.]

Le choix des valeurs usuelles mentionnées cidessus est actuellement controversé. Ainsi à l'aide d'un calcul Hartree-Fock pour un champ neutron de la région $\mathcal{A} \simeq 150$, Quentin [9] trouve l'ordre des orbites $h_{9 / 2}$ et $f_{7 / 2}$ inverse à celui des valeurs usuelles.

Une situation analogue se retrouve [10] dans la région $A \simeq 130$ pour les sous-couches $g_{7 / 2}$ et $d_{5 / 2}$ d'un champ neutron. Dans ces deux cas, on obtient pour les neutrons l'ordre des sous-couches habituellement adopté pour les protons.

Enfin, dans un article récent Rekstad [5] a montré que si on traitait correctement le terme de recul de l'hamiltonien, $\mu$ peut prendre des valeurs très différentes $(\mu=0,12,0,22,0,28, \ldots)$ de celles qu'on a 
habituellement pour un champ neutron dans la région des masses $A \simeq 150$.

Partant de ces données, nous avons effectué des calculs pour différentes valeurs de $\mu, \mathcal{A}$ et $\lambda$ pour $\mathrm{h}_{9 / 2}$ et $\mathrm{f}_{7 / 2}$ d'une part et $\mathrm{g}_{7 / 2}$ et $\mathrm{d}_{5 / 2}$ d'autre part. Il ressort de cette étude que lorsque la surface de Fermi $\lambda$ est placée soit au-dessus soit en dessous de l'ensemble de deux sous-couches, les résultats ainsi obtenus sont très proches de ceux obtenus avec les calculs n'introduisant qu'une seule sous-couche voisine et pour la même position de la surface de Fermi. On observe cependant que les niveaux sont un peu plus resserrés. Mais lorsque $\lambda$ se rapproche de l'ensemble des deux sous-couches, on observe nettement une plus forte densité de niveaux, le mélange des sous-couches étant plus fort.

Sur la figure 10 nous montrons le cas particulier où $\mu=0,50$ pour lequel les deux sous-couches $\mathrm{h}_{9 / 2}$ et $\mathrm{f}_{7 / 2}$ sont confondues à $\varepsilon=0$ (Fig. 9). Pour $\varepsilon<0$ on voit nettement deux bandes $1 / 2$ et $3 / 2$ et pour $\varepsilon>0$ on observe deux bandes $9 / 2$ et $7 / 2$ très rapprochées et presque confondues pour certaines déformations.

Il apparaît donc que la diagonalisation dans l'espace des deux sous-couches n'introduit pas des changements appréciables par rapport au traitement exact des énergies et des fonctions d'onde des états individuels.

5. Conclusion. - Nous avons étudié les limites d'application de l'hypothèse $\mathrm{j}=$ cte du modèle de Stephens et montré ses implications dans le cas des sous-couches non isolées. Un traitement exact des fonctions d'onde des états individuels, qui, soit dit en passant, ne présente aucune espèce de difficultés est cependant nécessaire lorsqu'on veut étendre le modèle de Stephens aux sous-couches non isolées. Dans le cas des sous-couches isolées $(N=3,4,5.6$ et 7), un tel traitement est suffisant, il est inutile d'étendre la base de diagonalisation.

La symétrie oblate-prolate obtenue en plaçant la

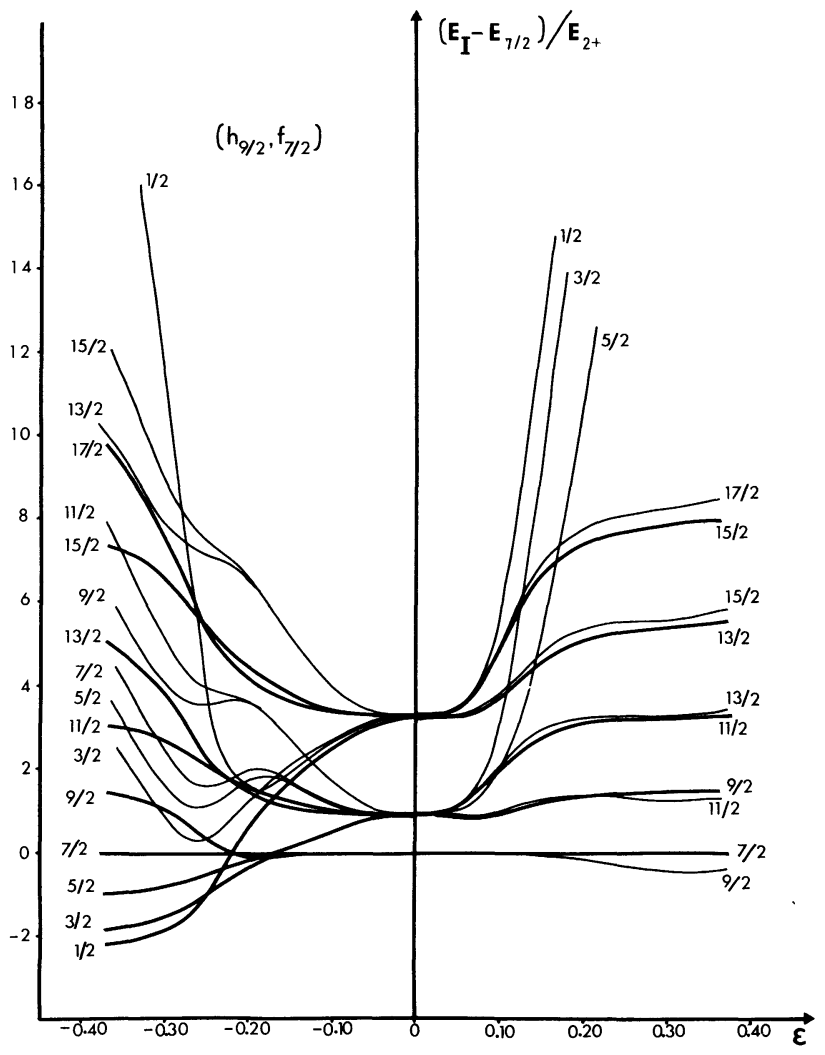

Fig. 10. - Résultats de la diagonalisation de l'éq. (1) pour différentes valeurs de $\varepsilon$, dans le cas de l'ensemble des deux sous-couches $\mathrm{h}_{9 / 2}$ et $\mathrm{f}_{7 / 2}, \lambda=7,20 \hbar \omega_{0}, \mathcal{A}=177, \mu=0,50$.

[Results of diagonalizing eq. (1) at various values of $\varepsilon$, for the system of both orbitals $\left(h_{9 / 2}, f_{7 / 2}\right) . \lambda=7.20 \hbar \omega_{0}, \mathcal{A}=177, \mu=0.50$.]

surface de Fermi au-dessus ou en dessous de la souscouche n'est cependant pas conservée pour des souscouches non isolées.

Enfin, il est peut-être utile d'ajouter que si on lève la symétrie axiale [11] on introduit naturellement des effets de mélange de sous-couches qui peuvent devenir importants et évidemment différents de ceux étudiés ici dans le cadre de l'hypothèse de la symétrie axiale.

\section{Bibliographie}

[1] Stephens, F. S., Rev. Mod. Phys. 47 (1975) 43.

[2] BoHr, A., Dan. Mat. Fys. Medd. 26 (1952) no 14.

[3] Nilsson, S. G., K. Dans. Vidensk. Selsk. Mat. Fys. Medd. 29 (1955) no 16.

[4] Lamm, I. L., Nucl. Phys. A 125 (1969) 504.

[5] Rekstad, J. et al., Phys. Lett. 62B (1976) 15 ; Nucl. Phys A 267 (1976) 40.

[6] Grodzins, L., Phys. Lett. 2 (1962) 88
[7] Meyer-Ter-Vehn, J., Nucl. Phys. A 249 (1975) 111 et 141.

[8] Boisson, J. P. et Piepenbring, R., Nucl. Phys. A 168 (1971) 385 .

[9] Quentin, P., Thèse Orsay (1975).

[10] Donau, F. et Hagemann, U., Nucl. Phys. A 256 (1976) 27.

111 Faessler, A. et ToKI, H., Phys. Lett. 59B (1975) 211.

[12] L $\varnothing$ vhøiden, G. et al., Nucl. Phys. A 148 (1970) 657. 